\title{
Analysis of impact of atmospheric refractive index and attenuation on la- ser energy transmission in near-Earth space
}

\author{
X. JIN \& Y. J. HONG \& D.P. WANG
}

State Key Laboratory of Laser Propulsion \& Application, Equipment Academy, Beijing, China

KEYWORD: Laser, Refractive index, Atmospheric molecules, Aerosols, Attenuation coefficient ABSTRACT: Based on background of laser atmospheric energy transmission to the UAV application, the impacts of atmospheric refractive on beam deflection is analyzed, and further the attenuation effects of atmospheric molecules and aerosols are discussed. Based on diffraction, turbulence, thermal blooming, optical system jitter and other divergent angle effects, namely the relationship between the optimum laser output beam region and the initial zenith angle, which provides design and analysis methods for UAV atmospheric energy transmission.

\section{Introduction}

Laser-motive UAV is a new kind of photovoltaic UAV. Compared to solar UAV, it has a large transmission power density, making the UAV with higher flight altitude, longer battery life and greater payload.

UAV works in near-Earth space flight altitude not exceeding $40 \sim 50 \mathrm{~km}$ range, where atmospheric refractive index, attenuation, diffraction, turbulence, optical system jitter and thermal blooming, etc., all have impacts on Laser energy transmission, namely have impacts on the UAV operating mode, thus analysis and study of near-Earth space laser energy transmission distribution characteristics can be of great significance $e^{[1-2]}$.

\section{UAVLight deflection caused by atmospheric refraction}

In the surface of earth, atmosphere refractive index distribution can be regarded as uniform radial and horizontal stratification, namely spherically symmetric. In symmetric central plane, the refractive index $n(r)$ is only relevant to the distance $r$ from the center of symmetry. When light passes through the atmosphere, the $\mathrm{Bu}$ Gaier formula is applicable, as shown below, where $\varphi$ is the angle between vector $\boldsymbol{r}$ and light path tangent (or light local zenith angle) ${ }^{[3]}$.

$n(r) r \sin \varphi=C($ cons $\tan t)$

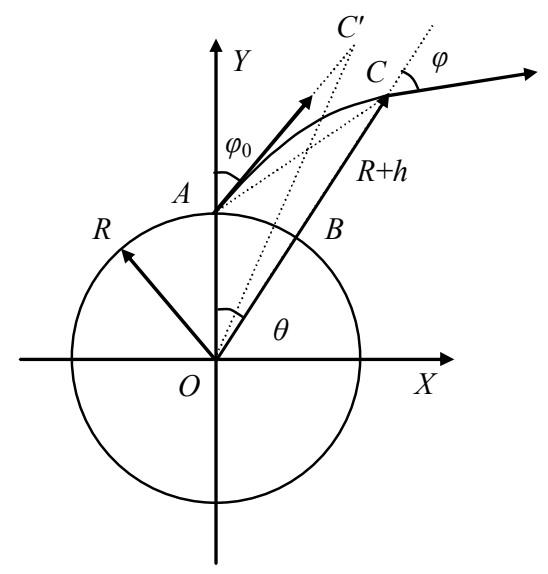

Figure 1. Deflection of light caused by refraction.

As is shown in Figure 1, the light trajectory equation is $r=r(\theta)$. For any central angular increments (clockwise from $Y$ axis is positive), the light arc trajectory meets Equation (2). 
$r(\theta) \mathrm{d} \theta=\mathrm{d} s \sin \varphi, \mathrm{d} s=\sqrt{r^{2}(\theta)+\left[\frac{\mathrm{d} r(\theta)}{\mathrm{d} \theta}\right]^{2}} \mathrm{~d} \theta$

thus:

$$
\sin \varphi=r(\theta) \frac{\mathrm{d} \theta}{\mathrm{d} s}=r(\theta) \frac{1}{\sqrt{r^{2}(\theta)+\left[\frac{\mathrm{d} r(\theta)}{\mathrm{d} \theta}\right]^{2}}}
$$

Combining Equation (3) and Equation (1) can get a result as below.

$$
\frac{\mathrm{d} r(\theta)}{\mathrm{d} \theta}=\frac{r(\theta)}{C} \sqrt{n^{2}(r) r^{2}(\theta)-C^{2}}
$$

Thus, if $r=R+h$ has incremental $\mathrm{d} r=\mathrm{d} h$, the corresponding central angle is shown in Equation (5) as below.

$$
\mathrm{d} \theta=\frac{C \mathrm{~d} h}{(R+h) \sqrt{n^{2}(h)(R+h)^{2}-C^{2}}}
$$

As shown in Figure 1, according to the initial boundary conditions of light incident point $A$, we can see $C=n_{0} R \sin \varphi_{0}$, where $n_{0}$ is the refractive index of the Earth's surface, $R=6378 \mathrm{~km}$ is the Earth's average radius, and $\varphi_{0}$ is the initial zenith angle of the light.

To any given ground level $h$, the corresponding central angle is given in Equation (6), and the corresponding ground arc distance $S_{A B}=A B=\theta R$.

$$
\theta=\int_{0}^{h} \frac{C \mathrm{~d} h}{(R+h) \sqrt{n^{2}(h)(R+h)^{2}-C^{2}}}
$$

According to boundary conditions of point C, $C=n_{0} R \sin \varphi_{0}=n(h)(R+h) \sin \varphi$ can be obtained, thus the zenith angle can be obtained as Equation (7).

$$
\sin \varphi=\frac{n_{0} R \sin \varphi_{0}}{n(h)(R+h)}, \varphi=\arcsin \frac{n_{0} R \sin \varphi_{0}}{n(h)(R+h)}
$$

The relative zenith angle from light tangent to initial position (Y-axis) is $\varphi+\theta$.

The zenith angle of the straight line $\mathrm{AC}$ between point $\mathrm{A}$ and point $\mathrm{C}$ is shown in Equation (8).

$$
\frac{\sin \left(\pi-\varphi_{01}\right)}{R+h}=\frac{\sin \theta}{A C}, \varphi_{01}=\arcsin \frac{(R+h) \sin \theta}{A C}
$$

In Equation (8),

$$
A C=\sqrt{R^{2}+(R+h)^{2}-2 R(R+h) \cos \theta}
$$

\section{Effects of atmospheric absorption and scattering}

Monochromatic light, with intensity of $I$, generates attenuation of $\mathrm{d} I$ after through the thin layer thickness $\mathrm{d} s$ as its thickness, in the atmosphere, which meets $\mathrm{d} I / I=-\beta \mathrm{d} s$, where $\beta$ is the attenuation coefficient, and the atmospheric transmittance (the optical intensity attenuation percentage in given transmission distance is shown as below.

$$
T=\frac{I}{I_{0}}=\exp \left(-\int_{0}^{L} \beta \mathrm{d} s\right)
$$

Atmospheric absorption and scattering attenuation contains two separate physical processes, thus the attenuation coefficient can be expressed as: 
$\beta=\beta_{m}+\beta_{a}=\left(k_{m}+\sigma_{m}\right)+\left(k_{a}+\sigma_{a}\right)$

where, $k_{m}$ and $\sigma_{m}$ are atmospheric absorption and scattering coefficients of molecules, and $k_{a}$ and $\sigma_{a}$ are atmospheric absorption and scattering coefficients of aerosols.

Atmospheric transmittance is

$T=\frac{I}{I_{0}}=\exp \left[-\int_{0}^{L}\left(\beta_{m}+\beta_{a}\right) \mathrm{d} s\right]=\exp \left(-\int_{0}^{L} \beta_{m} \mathrm{~d} s\right) \exp \left(-\int_{0}^{L} \beta_{a} \mathrm{~d} s\right)=T_{m} T_{a}$ Therefore, the impact of atmospheric mole-

cules and aerosols on attenuation can be calculated and analyzed separately ${ }^{[4]}$.

According to the hierarchical and horizontal radial uniformity hypothesis, the attenuation coefficient is a function of the ground level, and the height of the optical thickness of the ground is:

$\delta(h)=\int_{h}^{\infty} \beta \mathrm{d} h, \frac{\mathrm{d}[\delta(h)]}{\mathrm{d} h}=-\beta(h)$

The oblique light transmission distance is $\mathrm{d} s$, the strength of attenuation $\beta(h) \mathrm{d} s$, the corresponding height $\mathrm{d} h=\mathrm{d} s \cos \varphi$, so the atmospheric transmittance is:

$T=\frac{I}{I_{0}}=\exp \left[-\int_{0}^{L} \beta(h) \mathrm{d} s\right]=\exp \left[-\int_{0}^{h} \frac{\beta(h)}{\cos \varphi} \mathrm{d} h\right]$

According to $\mathrm{Bu}$ Gaier formula, after taking into account atmospheric refraction, Equation (13) is get.

$\int_{0}^{L} \frac{\beta(h)}{\cos \varphi} \mathrm{d} h=\int_{0}^{h} \frac{n(h)(R+h) \beta(h)}{\sqrt{n^{2}(h)(R+h)^{2}-C^{2}}} \mathrm{~d} h$

Obviously, deflection of light caused by atmospheric refraction also has effects on atmospheric attenuation. If the deflection angle caused by of the light refraction is relatively small, the effects of the refractive index can be ignored, the approximate formula is:

$\int_{0}^{L} \frac{\beta(h)}{\cos \varphi} \mathrm{d} h \approx \frac{1}{\cos \varphi_{0}} \int_{0}^{h} \beta(h) \mathrm{d} h$

\section{Laser Far field power density}

Define average laser power as $P$, the atmospheric attenuation coefficient as $\beta$, laser transmission distance (role distance) as $z$, the far-field laser power as $P_{s}=P \exp \left(-\int_{0}^{z} \beta \mathrm{d} s\right)$, far-field laser spot diameter as $w(z)$, then the far-field laser power density is:

$I_{s}=\frac{P_{s}}{A_{s}}=\frac{P \exp \left(-\int_{0}^{z} \beta \mathrm{d} s\right)}{\pi[w(z) / 2]^{2}}=\frac{4 P T}{\pi w^{2}(z)}$

For experimental convenience in engineering, the spot size is in accordance with $86.5 \%$ encircled energy definition, after $86.5 \%$ encircled energy corrected, the Gaussian beam far field spot diameter is contained in Equation (16).

$I_{s}=\frac{0.865 \times 4 P T}{\pi w^{2}(z)}$

Define laser beam waist diameter ( $z=0$ at the spot diameter) as $w_{0}$, divergence angle as $\sigma_{\text {total }}$, laser beam represented by hyperbolic is given in Equation (17).

$\frac{[w(z) / 2]^{2}}{\left(w_{0} / 2\right)^{2}}-\frac{z^{2}}{\left[\left(w_{0} / 2\right) / \tan \sigma_{\text {total }}\right]^{2}}=1, w(z)=w_{0} \sqrt{1+\left(\frac{\left.2 \tan \sigma_{\text {total }}\right)^{2}}{w_{0}} z^{2}\right.}$ 
where, $\sigma_{\text {total }}$ is the divergence angle (half size), including turbulence, heat faint, optical system jitter and other factors, as shown below.

$$
\sigma_{\text {total }}^{2}=\sigma_{\text {diffraction }}^{2}+\sigma_{\text {turbulence }}^{2}+\sigma_{\text {jitter }}^{2}+\sigma_{\text {bloom }}^{2}
$$

The divergence angle (half size) due to diffraction is:

$\sigma_{\text {diffraction }}=\frac{2}{\pi} \cdot \frac{B \lambda}{w_{0}}, \sigma_{\text {diffraction }}=\frac{2 B \lambda}{D}$

where, $w_{0}$ is Gaussian beam waist diameter; $B$ is the diffraction limit multiples ( laser beam quality). When the transmission aperture of the mirror is $D=\pi w_{0}$, laser energy through the aperture is more than $99 \%$.

Beam quality multiple of diffraction limit $2 \lambda /\left(\pi w_{0}\right)$ is shown as below.

$B_{S}=\sigma_{\text {total }} /\left[2 \lambda /\left(\pi w_{0}\right)\right]$

The laser density near field power is shown as below.

$I_{b}=\frac{0.865 \times 4 P}{\pi w_{0}^{2}}$

The relationship between the far field laser power density and near field power laser density is shown as below.

$I_{s}=T\left[\frac{w_{0}}{w(z)}\right]^{2} I_{b}, \frac{w(z)}{w_{0}}=\sqrt{1+\left(\frac{2 \tan \sigma_{\text {total }}}{w_{0}}\right)^{2} z^{2}}$

Obviously, the main factors affecting far field power density are: 1) the near field power density; 2) atmospheric transmittance; 3 ) laser beam expander.

\section{Atmospheric refractive index and attenuation index model}

If the refractivity model and attenuation index model are known, impacts of atmospheric attenuation coefficient and refractivity index can be analyzed. During laser energy transmission to air, the concern is spatial energy distribution energy attenuation caused, and therefore, the variation of atmospheric refractivity and atmospheric attenuation index with ground height should merely meet the basic variation ${ }^{[5]}$.

The relationship between refractivity modulus $N$ and refractive index $n$ is:

$N=(n-1) \times 10^{6}$

The relationship between $N, n$ and atmospheric pressure $p$ (unit: hPa), temperature $T$ (unit: $\mathrm{K}$ ), wavelength $\lambda$ (unit: $\mu \mathrm{m}$ ) is as below.

$N=77.6 \frac{P}{T}+0.584 \frac{P}{T \lambda^{2}}$

namely

$n=1+\left(77.6 \frac{P}{T}+0.584 \frac{P}{T \lambda^{2}}\right) \times 10^{-6}$

Marggrat and Griggs propose atmospheric molecular optical thickness in the vertical optical path as below

$\delta_{m}(h)=0.0088 \lambda^{-(4.15+0.2 \lambda)} \exp \left(-0.1188 h-0.00116 h^{2}\right) \quad 0.3 \leq \lambda \leq 10$

where, $h$ is height (unit: $\mathrm{km}$ ), $\lambda$ is wavelength (unit: $\mu \mathrm{m}$ ). It namely gives the atmospheric molecular attenuation index $\beta_{m}(h)=-\mathrm{d}\left[\delta_{m}(h)\right] / \mathrm{d} h$.

For empirical aerosol effect, empirical attenuation coefficient is shown as below. 
$\beta_{a}=\frac{3.912}{V_{M}}\left(\frac{0.55}{\lambda}\right)^{a} \exp \left(-\frac{h}{1.2}\right)$

where

$a=\left\{\begin{array}{l}1.6 \quad V_{M}>20 \mathrm{~km} \\ 1.3 \quad 6 \mathrm{~km} \leq V_{M} \leq 20 \mathrm{~km} \\ 0.585 V_{M}^{1 / 3} \quad V_{M}<6 \mathrm{~km}\end{array}\right.$

In the above, $h$ is the height (unit: $\mathrm{km}$ ), $\lambda$ is wavelength (unit: $\mu \mathrm{m}$ ) and $V_{m}$ is the visibility (unit: $\mathrm{km})$.

\section{Energy distribution of laser energy transmission in space}

Define laser wavelength as $\lambda=1.06 \mu \mathrm{m}$, visibility as $V_{m}=20 \mathrm{~km}$, the below discusses atmospheric attenuation distribution that atmospheric refractivity and attenuation caused.

As is shown in Figure 2, the ground aerosol attenuation coefficient $\beta_{a}$ is larger than atmospheric molecular attenuation coefficient $\beta_{m}$ two orders of magnitude, and effects of atmospheric molecules are negligible.

With the ground level increasing, the aerosol attenuation coefficient $\beta_{a}$ decreases rapidly. The two coefficients are substantially the same at a height of about $5.3 \mathrm{~km}$, but attenuation caused by atmospheric molecular is dominant if height continues to increase.

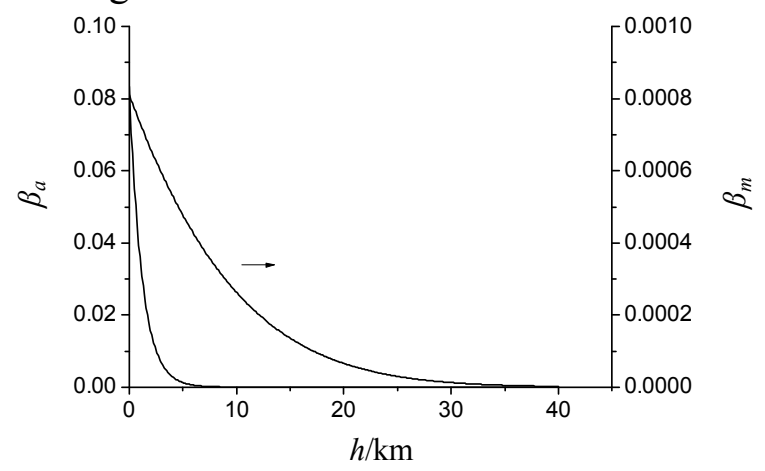

Figure 2. Aerosol attenuation coefficient varies with high variation.

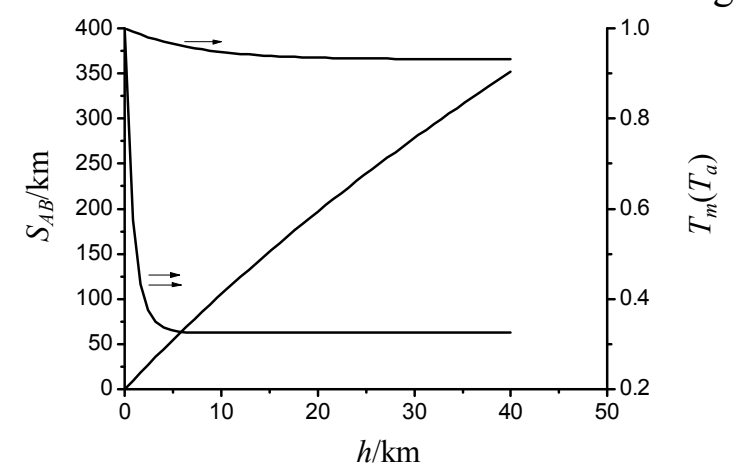

Figure 3. Atmospheric transmittance ground level with height and distance variation.

The greater of the light's initial zenith angle $\varphi_{0}$, the greater of the slant path (or ground level distance) achieved in the same height, and the greater of the refractive index and attenuation impacts. As shown in Figure 3, $\varphi_{0}=85^{\circ}$, the height is $40 \mathrm{~km}$, and the distance from the ground level $S_{A B}$ is up to $350 \mathrm{~km}$ or more. The impact of aerosols on transmittance $T_{a}$ (double arrow) works at a height of $5 \sim 6 \mathrm{~km}$ or less, and then levels off. The impact of atmospheric molecules affect transmittance $T_{m}$ (single arrow), mainly in height $5 \sim 6 \mathrm{~km}$ above (and relatively small). So in the mountains and highland areas, by reducing the aerosol effect, laser energy transmission can exhibit greater atmospheric transmittance.

As shown in Fig 4, $\varphi_{0}=85^{\circ}$, by comparison of the relative error in Equation (14) and Equation (13), in the range of $\varphi_{0}=0^{\circ} \sim 85^{\circ}$, the relative error of molecular transmission calculated by Equation (14) is less than $1 \%$, and the relative error of aerosol transmission is less than $3 \%$. Namely in the 
range of $\varphi_{0}=0^{\circ} \sim 85^{\circ}$ and the ground height less than $40 \mathrm{~km}$, the calculation error is less than $5 \%$ if the approximate formula applied, basically meeting the requirements of engineering calculations.

As shown in Fig 5, $\varphi_{0}=45^{\circ}$, as height increases, the light zenith angle $\varphi_{01}$ and the light deflection angle $\varphi+\theta$ are gradually increased. In the range of $\varphi_{0}=0^{\circ} \sim 45^{\circ}$ and the ground height less than $40 \mathrm{~km}$, the light deflection angle $\varphi+\theta<0.02^{\circ}$, and it is stabilized at the height above $30 \mathrm{~km}$.

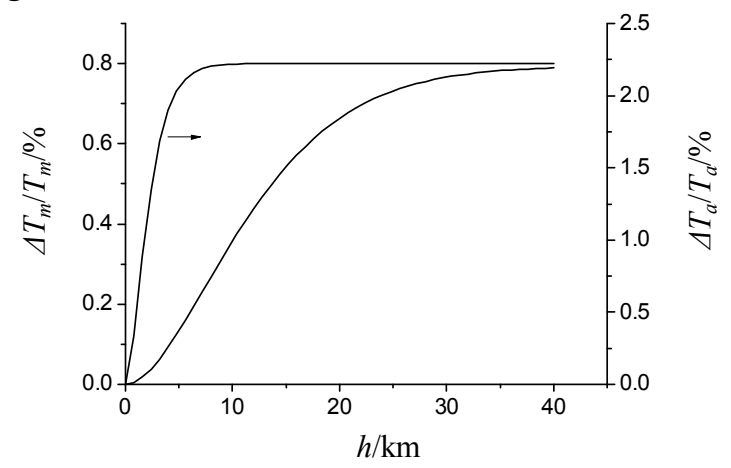

Figure 4. Approximate formula error varies with height.

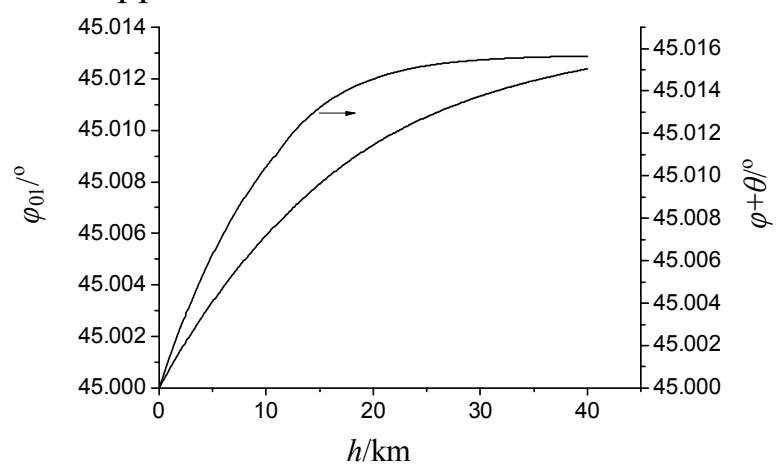

Figure 5. Light zenith angle and deflection angle varies with height.

The atmospheric transmittance with light initial zenith and elevation changes is shown in Figure 6. In the range of $\varphi_{0}=40^{\circ} \sim 50^{\circ}$, the atmospheric transmittance is greater, and more laser transmission energy can be obtained.

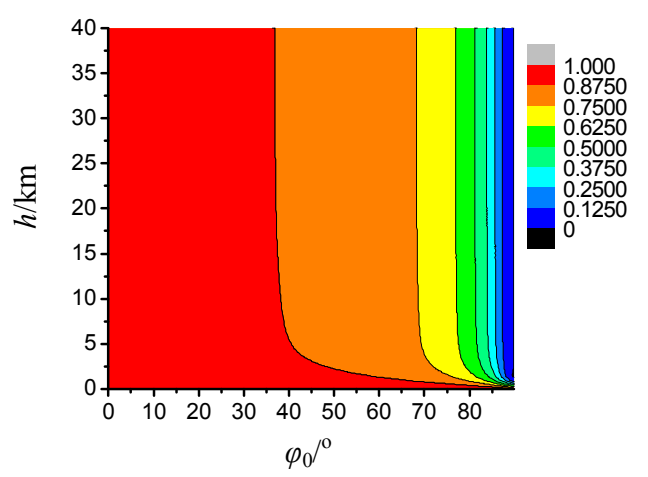

Figure 6. Atmospheric transmittance with initial zenith angle and height changes.

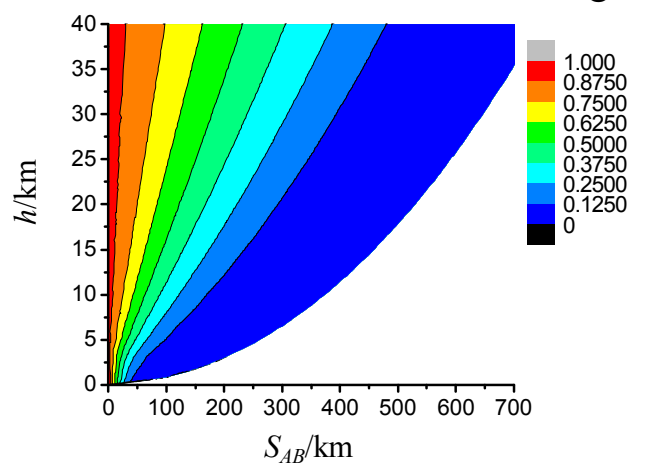

Figure 7. Atmospheric transmittance with the horizontal distance and height changes 
Figure 7 shows atmospheric transmittance initial zenith angle and height changes. Atmospheric transmittance is in cone distribution: greater than 0.5 in the ground level height of less than $40 \mathrm{~km}$ and horizontal distance $200 \mathrm{~km}$, and with greater availability of laser energy transmission of in the region.

Currently, for the typical kilowatt order optical fiber lasers with $\lambda=1.06 \mu \mathrm{m}$, the laser beam quality is within $B=3 \sim 35$ range. The upper limit of the divergence angle is $\sigma_{\text {turbulence }}=10 \mu \mathrm{rad}$ (turbulence), $\sigma_{\text {jitter }}=6 \mu \mathrm{rad}$ (foundation platform jitter), $\sigma_{\text {bloom }}=1 \mu \mathrm{m}$ (thermal blooming).

As shown in Fig 8, with the multiple of laser diffraction limit $B$ increase, diffraction makes divergence angle increases (left under the curve), and turbulence, diffraction, heat-blooming, platform jitter and total divergence angle increase, and $\sigma_{\text {diff }} / \sigma_{\text {total }}$ closes to 1 when $B \geq 15$, which indicates that at this time the diffraction divergence angle causes the greatest contribution to the total divergence angle. Figure 9 shows the total beam quality $B_{S}=\sigma_{\text {total }} /\left[2 \lambda /\left(\pi w_{0}\right)\right]$ changes with laser beam quality $B$, and they are basically equal when $B \geq 15$.

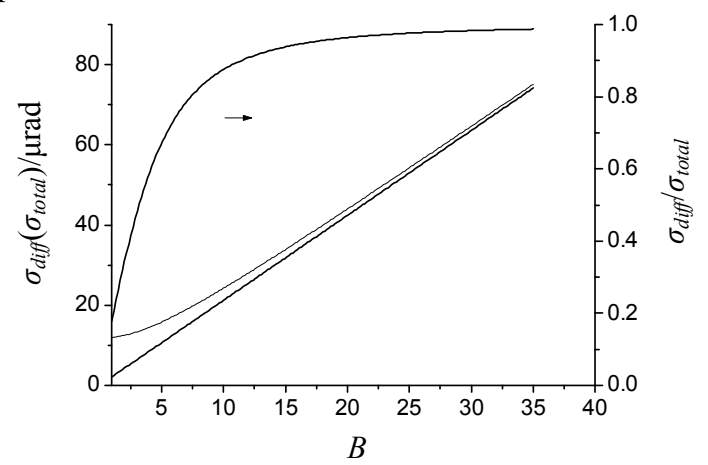

Figure 8. Atmospheric transmittance with initial zenith angle and height changes.

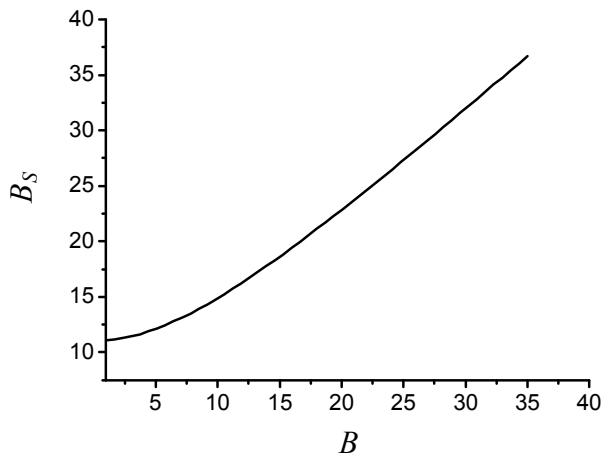

Figure 9. Atmospheric transmittance with initial zenith angle and height changes.

As shown in Fig 10, as the laser beam quality and transmission distance increases, the far field spot diameter increases, which results in the far field power density decreases rapidly. As shown in Figure 11 , the diameter of emission mirrors from $D=1 \mathrm{~m}$ turning to $D=1.5 \mathrm{~m}$, the far field spot diameter significantly reduces. Obviously, improving laser beam quality and increasing the laser beam emission mirror diameter can effectively reduce the far field spot diameter and enhance far field power density.

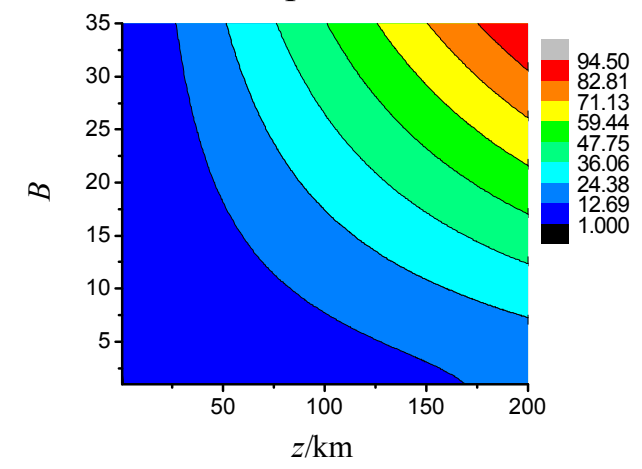

Figure 10. Atmospheric transmittance with initial zenith angle and height changes. 


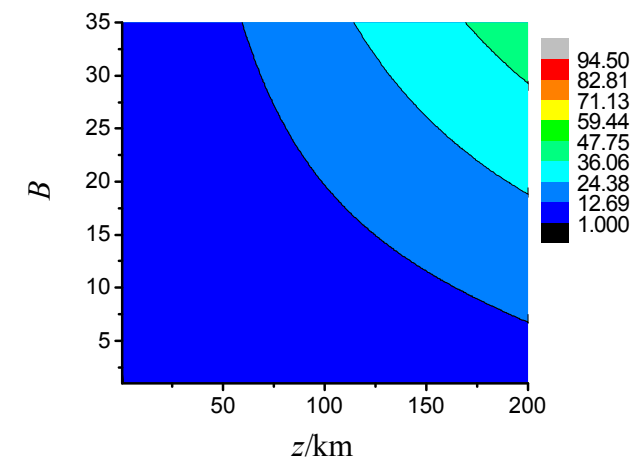

Figure 11. Atmospheric transmittance with initial zenith angle and height changes.

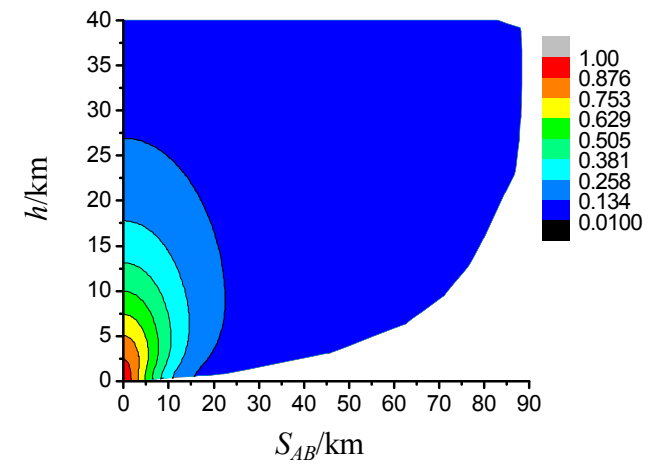

Figure 12. Atmospheric transmittance with initial zenith angle and height changes.

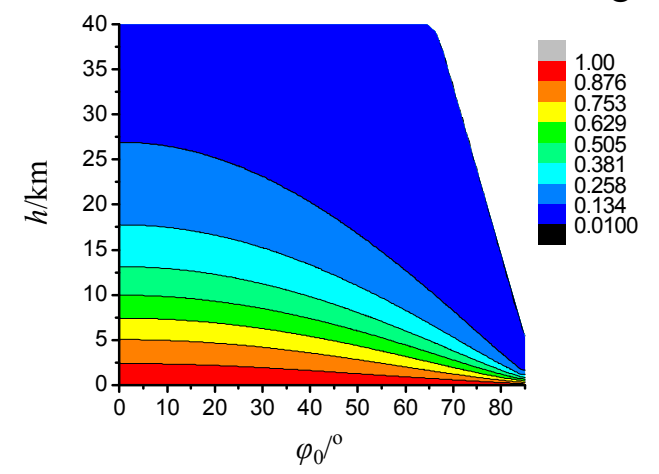

Figure 13. Atmospheric transmittance with initial zenith angle and height changes.

Figure 12 and Figure 13 separately shows the variation of far-field and near-field power density ratio $I_{s} / I_{b}$ when $\mathrm{D}=1.5 \mathrm{~m}$ and $\mathrm{BS}=15$. The isosurface of laser energy transmission is pear-shaped, and the width of the surface is less than height (shown in Figure 13, $I_{s} / I_{b}$ is reduced with the initial zenith angle increases).

\section{Conclusions}

The laser energy transmission method can provide laser driven UAV with energy. It is needed to research the laser energy transmission strategies according to the distribution of refractive index and attenuation coefficient, and conclusions are as following:

(1)Aerosols play a leading role to influencing transmittance below, while atmospheric molecular do above ground level $5 \sim 6 \mathrm{~km}$, and aerosols make greater contribution to attenuation on the whole. Consequently, in the mountains and highland areas, reducing the aerosol effects can acquire greater availability of atmospheric transmittance, which is beneficial to laser energy transmission.

(2)According to the atmospheric transmittance variation as initial light zenith angle and height, we can see that atmospheric transmittance is greater in the range of $\varphi_{0}=0^{\circ} \sim 50^{\circ}$ and greater availability of larger energy transmission can be get, namely within the $\varphi_{0}=0^{\circ} \sim 50^{\circ}$ cone area being the optimum laser emission angle.

The isosurface of laser energy transmission is pear-shaped, and the width of the surface is less than height. Furthermore, improving laser beam quality and increasing the laser beam emission mirror diameter can effectively reduce the far field spot diameter and facilitate laser energy transmission. 
In short, according to the energy distribution characteristics in spatial, it is feasible to drive laser powered UAV through the atmosphere.

\section{REFERENCES}

[1] Myrabo L N. Propulsion systems integration for a 'tractor beam' mercury lightcraft:liftoff engine [A]. First International Symposium on Beamed Energy Propulsion [C], 2003, 683-694.

[2] Edward H. Allen. The case for near space [J]. Aerospace America, 2006, (2):31-34.

[3] JoeT.Howell,MarkJ.Oneill,RichardL.Fork.Ad-vanced receiver/converter experiments for laser wireless power transmission [A]. Solar Power from Space and 5th Wireless Power Transmission Conference [C], 2004.

[4] Jin X, Chang H, Cui X Y. Concept research of laser-motive UAV [J]. Acta Aeronautica et Astronautica Sinica, 2013, 34(9):2074-2080.

[5] Junshu $\mathrm{Xu}$, et al. Diode large-signal characteristics measurement for high-power rectennas [J]. Microwave and Optical Technology Letters, 2005 45(3): 249-251. 